\title{
Dominant and recessive inheritance of morbid obesity associated with melanocortin 4 receptor deficiency
}

\author{
I. Sadaf Farooqi, ${ }^{1}$ Giles S.H. Yeo, ${ }^{1}$ Julia M. Keogh,${ }^{1}$ Shiva Aminian, ${ }^{1}$ Susan A. Jebb,${ }^{2}$ \\ Gary Butler, ${ }^{3}$ Tim Cheetham, ${ }^{4}$ and Stephen O’Rahilly ${ }^{1}$
}

1University Departments of Medicine and Clinical Biochemistry, Addenbrooke's Hospital, Cambridge, United Kingdom
${ }^{2}$ Medical Research Council, Human Nutrition Research, Cambridge, United Kingdom
${ }^{3}$ Leeds General Infirmary, Leeds, United Kingdom
${ }^{4}$ Royal Victoria Infirmary, Newcastle, United Kingdom

Address correspondence to: Stephen O'Rahilly, University Departments of Medicine and Clinical Biochemistry,

Box 157, Addenbrooke's Hospital, Cambridge CB2 2QR, United Kingdom. Phone: 44-1223-336855;

Fax: 44-1223-330598; E-mail: sorahill@hgmp.mrc.ac.uk.

I. Sadaf Farooqi and Giles S.H. Yeo contributed equally to this work.

Received for publication January 17, 2000, and accepted in revised form May 2, 2000.

\begin{abstract}
Over 20 severely obese subjects in 11 independent kindreds have been reported to have pathogenic heterozygous mutations in the gene encoding the melanocortin 4 receptor (MC4R), making this the most common known monogenic cause of human obesity. To date, the detailed clinical phenotype of this dominantly inherited disorder has not been defined, and no homozygous subjects have been described. We determined the nucleotide sequence of the entire coding region of the MC4R gene in 243 subjects with severe, early-onset obesity. A novel two-base pair GT insertion in codon 279 was found in two unrelated subjects, and four novel missense mutations, N62S, R165Q, V253I, C271Y, and one mutation (T112M) reported previously were found in five subjects. N62S was found in homozygous form in five children with severe obesity from a consanguineous pedigree. All four heterozygous carriers were nonobese. Several features of the phenotype, e.g. hyperphagia, tendency toward tall stature, hyperinsulinemia, and preserved reproductive function, closely resemble those reported previously in $M c 4 r$ knock-out mice. In addition, a marked increase in bone mineral density was seen in all affected subjects. In transient transfection assays, the N62S mutant receptor showed a responsiveness to $\alpha \mathrm{MSH}$ that was intermediate between the wild-type receptor and mutant receptors carrying nonsense and missense mutations associated with dominantly inherited obesity. Thus $M C 4 R$ mutations result in a syndrome of hyperphagic obesity in humans that can present with either dominant or recessive patterns of inheritance.
\end{abstract}

J. Clin. Invest. 106:271-279 (2000).

\section{Introduction}

The importance of central melanocortinergic pathways in the control of mammalian energy homeostasis has been highlighted recently by genetic studies in obese humans and mice. Disruption of the pro-opiomelanocortin gene in mice (1) and humans (2) results in an obese phenotype, as does the overexpression of the melanocortin antagonists agouti and agouti-related protein (3). Additionally, murine and human obesity syndromes resulting from mutations in enzymes involved in propeptide processing are associated with markedly impaired processing of pro-opiomelanocortin (POMC) $(4,5)$. Of the known receptors for melanocortin peptides only two, melanocortin 3 receptor (MC3R) and $\mathrm{MC} 4 \mathrm{R}$, are highly expressed in the central nervous system $(6,7)$. The critical role for the MC4 receptor in energy balance was first demonstrated by Huszar et al. with their observation that mice with null mutations in the MC4R gene developed hyperphagia, obesity, and hyperinsulinemia (8). Of particular note was the fact that heterozygous animals had a body weight intermediate between wild-type and homozygous-null littermates.
The importance of the MC4R in the regulation of human body weight first became apparent in 1998 with the reports from our group (9) and Vaisse et al. (10) of single families in whom heterozygous frameshift mutations in MC4R cosegregated with dominantly inherited severe early-onset obesity. Subsequent to these reports $\mathrm{Gu}$ et al. (11) reported two missense mutations in 140 obese subjects and Hinney et al. (12) reported nine missense mutations and two nonsense mutations in 306 obese children. Several conservative amino acid changes have been reported in more than one study. Notwithstanding the uncertainties regarding pathogenic mutations versus incidental amino acid changes, at least 20 subjects worldwide have, to date, been reported to have presumed pathogenic mutations in the MC4R gene, making it the most common monogenic form of human obesity described. Thus far, all affected subjects described have been heterozygotes.

The $M c 4 r$ knock-out (Mc4r-KO) mouse has a number of notable phenotypic features. In contrast to the $o b / o b$ and $d b / d b$ mice, $M c 4 r$-KO mice have normal reproductive function and increased, rather than decreased, lin- 
ear growth. Mc4r-KO mice do, however, show the hyperinsulinemia that is characteristic of many murine models of obesity. The published phenotypic data on MC4Rdeficient human subjects is very limited, restricted in most cases to simple anthropometric measures. We have undertaken further studies to better define the role of MC4R deficiency in human early-onset obesity. We have determined the frequency of $M C 4 R$ mutations in a large population of severely obese subjects and report the first pedigree containing homozygous mutant subjects. We report the detailed clinical phenotype of nine affected subjects from three different pedigrees including measurements of body composition, energy expenditure, intake at a test meal, and endocrine physiology. Finally, our studies of the in vitro signaling properties of missense and nonsense mutations associated with severe obesity in the heterozygous or homozygous state provide some clues to the molecular basis for a genotype/phenotype relationship.

\section{Methods}

Subjects. Two hundred and forty-three unrelated U.K. probands were examined for mutations at the MC4R locus. All subjects had developed severe obesity before the age of 10 years. The mean body mass index (BMI; weight in kilograms divided by height in meters squared) standard deviation score (SDS) at presentation to the study was 4.17. All studies were approved by the local ethical committee and were conducted in accordance with Declaration of Helsinki principles. To facilitate better interpretation of the potential pathogenicity of any mutation found, the entire MC4R sequence was determined in 54 healthy nonobese, British Caucasian subjects.

Direct nucleotide sequencing of MC4R gene. Two primers, MC4Rforward (5'-AATAACTGAGACGACTCCCTGAC-3') and MC4Rreverse (5'-CAGAAGTACAATATTCAGGTAGGG$\left.3^{\prime}\right)$, were used in a PCR reaction to amplify the MC4R gene from genomic DNA isolated from whole blood. The PCR was performed using BioTaq (Bioline, London, United Kingdom) and carried out under standard conditions, with 35 cycles of $95^{\circ} \mathrm{C}$ for 30 seconds, $57^{\circ} \mathrm{C}$ for 30 seconds, and $72^{\circ} \mathrm{C}$ for 50 seconds. Six nested primers, MC4F1 (5'-TGAGACGACTCCCTGACCCAG-3'), MC4F2 (5'-CATCACCCTATTAAACAGTACAG-3'), MC4F3 (5'-AGGCTTCACATTAAGAGGATTG-3'), MC4R1 (5'TACAATATTCAGGTAGGGTAAGA-3'), MC4R2 (5'-TTGGCGGATGGCACCAGTGC-3'), and MC4R3 (5'-CACTGTGAAACTCTGTGCATC-3') were then used at an annealing temperature of $57^{\circ} \mathrm{C}$ to sequence the resulting PCR product on both strands. Sequencing was carried out using Big Dye terminator chemistry (Perkin-Elmer, Foster City, California, USA) and electrophoresed on an ABI 377 automated DNA sequencer. Sequences were assembled and examined using Sequencher software (Gene Codes, Ann Arbor, Michigan, USA).

Phenotypic studies. All subjects were assessed and examined by the same physician (I.S. Farooqi), and written consent for these studies was obtained. Height was measured to the nearest $0.5 \mathrm{~cm}$ using a portable free- standing stadiometer (Leicester Height Measure, London, United Kingdom). Weight was measured in the fasting state on a digital electronic balance accurate to the nearest $100 \mathrm{~g}$ (Fereday \& Sons 824/890, London, United Kingdom). Blood pressure was measured in the rested, fasted state. Body composition was measured using whole-body dual-energy $x$-ray absorptiometry (DXA) to determine bone mineralization, total lean mass, and total fat mass (DPX software; Lunar Corp., Madison, Wisconsin, USA). All children underwent a radiograph of the left hand and wrist for bone-age determination; films were read by the same radiologist (using the Greulich and Pyle method), who was blinded as to subject classification, pubertal stage, and age.

The basal metabolic rate (BMR) was measured by indirect calorimetry using an open-circuit, ventilated, canopy measurement system (Europa Gas Exchange Monitor; Nutren Technology Ltd., Manchester, United Kingdom). The measurement was conducted under standard conditions; subjects were lying at complete rest after an overnight fast and after sleeping for a minimum of 8 hours in a thermoneutral environment. For Caucasian adults (subjects 1, 2, 4, 5), measured BMR was compared to BMR predicted by age and gender-specific equations for North Europeans and Americans derived by Hayter and Henry, which are based on body weight (13). Because these equations overestimate BMR in some ethnic groups (by about 10\%), predicted BMR for adults of Indian/Pakistani origin was calculated using equations for Indian males (14) and females (15). For children, BMR was predicted on the basis of gender, height, and weight, using equations derived from a large study of obese and nonobese children by Molnar et al. (16).

A semiquantitative assessment of ingestive behavior was undertaken in children under the age of 18 years. An ad libitum meal of fixed size and content (18 MJ) was provided at breakfast after an overnight fast. The contents were covertly weighed before and after consumption and total energy intake and nutrient composition calculated using standard tables (17). In addition, energy intake was expressed per kilogram of lean body weight as a means of comparing intake between subjects of different age and body size.

Metabolic and endocrine tests. Blood samples were obtained in the fasting state and analysed for leptin, cholesterol, triglycerides, HDL cholesterol, LDL cholesterol, glucose, insulin, proinsulin, and split 32/33 proinsulin. Serum leptin levels were determined using a commercially available kit (Linco Research, Inc., St. Charles, Missouri, USA). Thyroid-stimulating hormone, free thyroxine, adrenocorticotrophin hormone (ACTH), follicle-stimulating hormone (FSH), luteinizing hormone (LH), estradiol, and testosterone were measured using standard assays. A 24-hour urine collection was obtained on some subjects and urine free cortisol measured.

In vitro function of mutant receptors. Mutant $M C 4 R$ s were amplified directly from patient DNA using primers 
MC4Rforward (see above) and MC4RrevEco (5'-CGCTTAAGTTAATATCTGCTAGACAAGTCAC-3'). MC4Rforward flanks an endogenous EcoRI site, whereas MC4RrevEco contains a terminal EcoRI restriction site, thus facilitating cloning of the PCR products into the mammalian expression vector pcDNA3 (Invitrogen Corp., San Diego, California, USA). PCR was performed using Expand DNA polymerase (Roche Diagnostics Corp., Indianapolis, Indiana, USA), according to manufacturer's protocols. All cloned products were sequenced as described above. HEK293 cells were maintained in DMEM (Life Technologies, Rockville, Maryland, USA) supplemented with 10\% FBS, $100 \mathrm{U} / \mathrm{mL}$ penicillin, $100 \mu \mathrm{g} / \mathrm{mL}$ streptomycin, and $250 \mathrm{ng} / \mathrm{mL}$ Fungizone (Life Technologies). Cells were incubated at $37^{\circ} \mathrm{C}$ in humidified air containing $5 \% \mathrm{CO}_{2}$, and transfections were carried out using Fugene reagent (Roche Diagnostics Corp.) according to manufacturer's protocols. Cells were generally at $70-80 \%$ confluence on the day of transfection. The cAMP responsive luciferase construct (LUC) was used here as described previously (18): $300 \mathrm{ng}$ of wild-type or mutant MC4R expression vector was transfected with 60 ng LUC reporter construct and $20 \mathrm{ng}$ of internal control plasmid, pRL-CMV (Promega Corp., Madison, Wisconsin, USA), which constitutively expresses Renilla luciferase. After transfection, cells were serum starved for 8 hours. Varying concentrations of $\alpha \mathrm{MSH}$ [Bachem (UK) Ltd., Saffron Walden, United Kingdom] were then added to the cells and allowed to incubate for 16 hours. The cells were lysed, and the two different luciferases were sequentially activated and quenched using the DualLuciferase Reporter Assay System (Promega) and the resulting luminescence detected using an AutoLumat LB953 luminometer (EG\&G Berthold, Baqd Wildbad, Germany) according to manufacturers' protocols.

\section{Results}

Identification of MC4R mutations. In the cohort of 243 unrelated subjects with severe early-onset obesity, no subject had the CTCT deletion at codon 211 that had been described previously by us in a British family. The sequence variants detected in this study and their location in the MC4R protein structure are described in Table 1 and Figure 1, respectively. A novel frameshift mutation consisting of a di-nucleotide (GT) insertion at codon 279 was found in two unrelated probands. Seven different point mutations that result in a change of amino acid sequence were found. T112M, R165Q, V253I, and C271Y were each found as heterozygous changes in single probands. N62S was found as a homozygous mutation in one proband. Other than T112M, which was reported previously in both a lean (11) and an obese subject (12), all the above mutations are novel. None of the above variants were found in 108 alleles from nonobese U.K. Caucasian controls. Two previously described sequence variants were found: V103I in three and I251L in seven subjects, respectively. These two variants were found at allelic frequencies of $0.9 \%$ and $2.7 \%$, respectively, in our Caucasian control population. We also identified an obese proband who was heterozygous for a deletion of a $\mathrm{C}$ nucleotide in the $3^{\prime}$ untranslated region, 28-base pairs downstream of the stop codon.

Family members were available for study in the case of three of the above probands (Figure 2). Also included in Figure 2 is the family tree of the subject with the CTCT deletion reported by us previously (9) because phenotypic data on this family will be described further. In the case of the GT insertion, this was transmitted to the proband by her affected father, who also had severe early-onset obesity, whereas the lean mother was wildtype at this locus. The C271Y mutation also appears to segregate with obesity in a dominant fashion. N62S was found in homozygous form in five children with severe obesity from a highly consanguineous extended family of Pakistani origin. In this pedigree, none of the four heterozygote parents had a body mass index of 30 $\mathrm{kg} / \mathrm{m}^{2}$ or more. Notably, this is the first description of homozygous $M C 4 R$ mutations in human subjects and of a recessive pattern of inheritance for mutations in this gene. Finally, the family of one subject with the common V103I variant was studied, and there was evidence against cosegregation of this variant with obesity, which is consistent with the reported normal signaling properties of this mutant in vitro $(11,19)$.

Phenotypic studies. Affected and unaffected members from three families in whom mutations in MC4R cosegregate with obesity (CTCT $\Delta$, GTins, and N62S) were subjected to a more-detailed evaluation to better define the clinical, metabolic, and endocrine phenotype of human MC4R deficiency. By definition, all probands were obese from an early age. For the purposes of further discussion, the term "affected" refers to heterozygotes in the case of the frameshift mutations and homozygotes in the case of the N62S mutation. In total, four affected adults (two heterozygous frameshift and

\section{Table 1}

Sequence variants of MC4R detected in 243 subjects with severe early-onset obesity

$\begin{array}{lccc}\text { Sequence variant } & \begin{array}{c}\text { Number of } \\ \text { obese subjects } \\ \text { with mutation }^{\mathrm{A}}\end{array} & \begin{array}{c}\text { Number of } \\ \text { controls } \\ \text { with mutation }\end{array} & \begin{array}{c}\text { Number of } \\ \text { subjects } \\ \text { previously } \\ \text { described }\end{array} \\ \begin{array}{l}\text { GT insertion at codon 279 } \\ \text { C deletion 28-bp }\end{array} & 2 & 0 & \text { None } \\ \text { downstream of stop codon } & 1 & 0 & \text { None } \\ \text { N62S } & 1 \text { (hom) } & 0 & \text { None } \\ \text { T112M } & 1 & 0 & 2(11,12) \\ \text { R165Q } & 1 & 0 & \text { None } \\ \text { V253I } & 1 & 0 & \text { None } \\ \text { C271Y } & 1 & 0 & \text { None } \\ \text { I251L } & 7 & 3 & 1(12) \\ \text { V103I } & 3 & 1 & 2(11,12)\end{array}$

ATwo hundred forty-three severely obese subjects were studied. All sequence variants detected were heterozygous except for N62S, which was found in homozygous form (hom). ${ }^{B}$ Fifty-four healthy nonobese U.K. Caucasians were studied. 


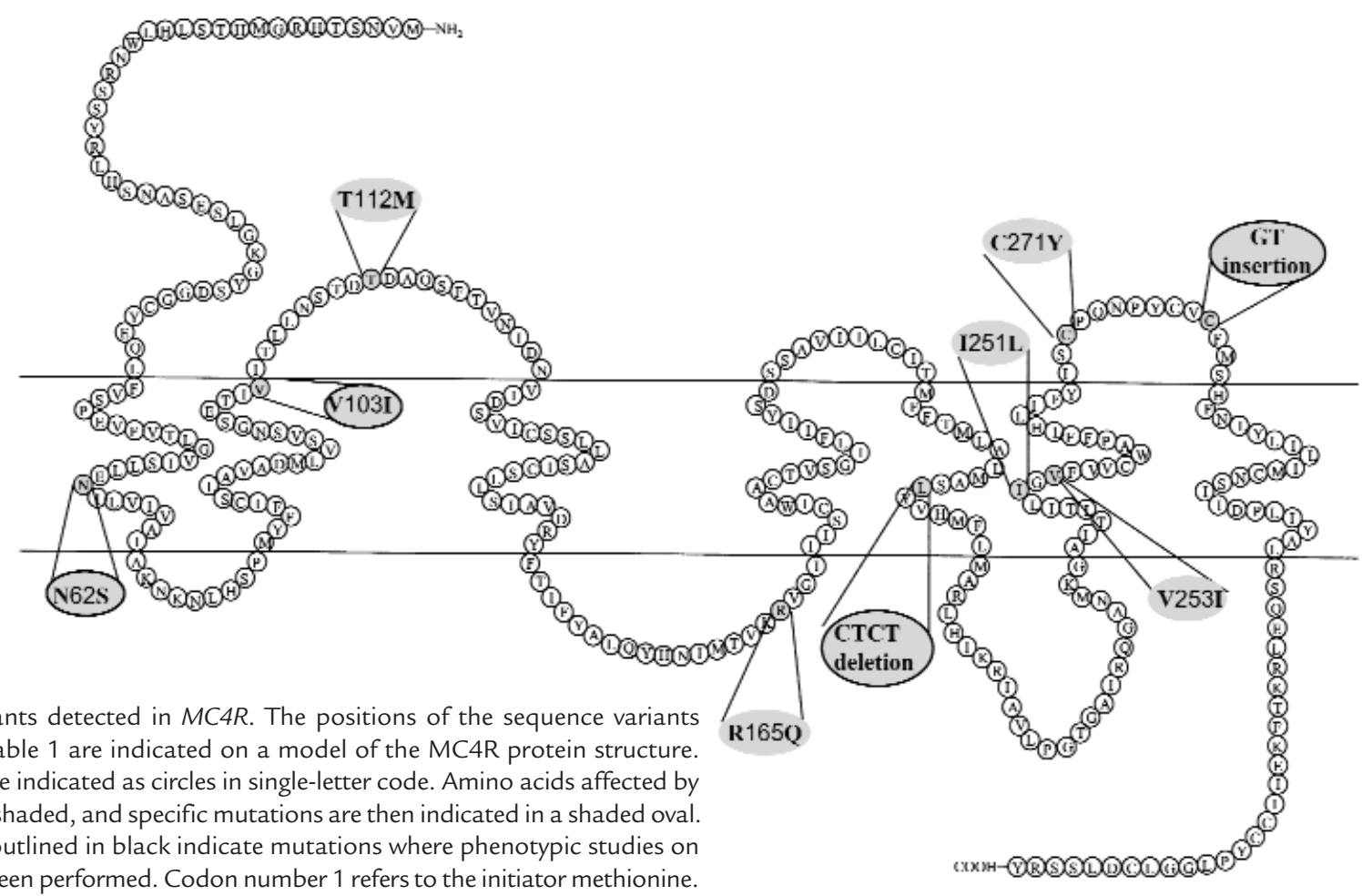

two homozygous N62S) and five affected children (two heterozygous frameshift and three homozygous N62S) were studied. The nonobese subjects from these families that were studied comprised two wild-type and four heterozygous N62S adults and two wild-type children.

Table 2 describes the clinical features and results of anthropometry, body composition, basal energy expenditure, and energy intake measurements of all subjects in these three families. In all cases the parents reported affected children to be excessively hungry from 6-8 months of age, with persistent food-seeking behavior and distress if food was not provided. Apart from obesity and hyperphagia, the affected children had no other developmental, intellectual, or behavioral problems. On clinical examination, apart from obesity, there were no dysmorphic features. No subject had acanthosis nigricans. Blood pressure was elevated in two of four affected adults and systolic blood pressure was greater than the 95th percentile for age, gender, and height in two of five affected children. Of note, however, two of four nonobese heterozygous adults were markedly hypertensive. Body temperature was normal in all subjects. Given the increased linear growth of $M c 4 r$-KO animals, it is of note that the affected children were on average on the 91st percentile for height. In the N62S family, in particular, the two homozygous wild-type children (subjects 11 and 15) are on the ninth and 25th percentiles for height, respectively, whereas the homozygous mutant children are on the 91st-99th percentiles for height. Growth charts available on several affected subjects confirmed an increased growth velocity in childhood (data not shown). Bone age was assessed by radiography of the wrist in all children in these pedigrees. Bone age exceeded chronological age in four of five affected children by a range of 1.0-4.9 years.

Body composition was assessed by DXA scanning. Of particular note is the finding that total bone mineral density (BMD) in affected subjects was consistently greater than expected from the age-adjusted population range $(Z$ score, mean \pm SD: $2.32 \pm 0.69)$. In contrast, the total body BMD measurements in nonobese subjects from the three families were similar to age- and genderrelated reference values $(Z$ score, mean \pm SD: $0.72 \pm$ 1.16). Total body bone mineral content (BMC) was also increased in all affected subjects compared with ageand gender-specific data (20).

BMR was measured in all subjects by indirect calorimetry. In the case of the Caucasian subjects with heterozygous frameshift mutations, there was no significant deviation of these measurements from those predicted by the Hayter and Henry equations relating BMR to body weight (13). In contrast, all adult subjects in the N62S family (unaffected and affected) had BMRs considerably lower than that predicted by the same equation (data not shown), which may reflect ethnic differences reported previously (21). Predicted BMRs for adults of Pakistani origin (subjects 7-10, 13, and 14) were obtained using equations derived from two different data sets based on males and females living in India $(14,15)$. Compared with these predictions, all four nonobese adults and two affected adults had measured BMRs only slightly below predicted levels. Measured BMRs for affected children were similar to values predicted on the basis of age, gender, height, and weight. 
Given the limitations of methods used to assess energy intake in free-living humans, we undertook semiquantitative studies of ingestive behavior in all children in these families. A large test meal of known nutrient and energy content was provided after an overnight fast, and the quantity and macronutrient composition of the food ingested was recorded. All affected subjects had greatly increased energy intake when compared with wild-type siblings (mean for affected subjects 4.1 MJ and mean for unaffected subjects $1.9 \mathrm{MJ}$ ). When energy intake was expressed as kilojoules per kilogram of lean body mass (as an approximate adjustment for the body size of the subjects studied), the mean energy intake of affected subjects was observed to be more than twice that of unaffected subjects $(124 \mathrm{~kJ} / \mathrm{kg}$ and $50 \mathrm{~kJ} / \mathrm{kg}$, respectively; unpaired $t$ test: $P<0.05)$. There was no evidence for macronutrient preference between affected subjects $(65 \%$ carbohydrate, $15 \%$ protein, $20 \%$ fat) and wild-type siblings (72\% carbohydrate, $10 \%$ protein, $18 \%$ fat).

Secondary sexual characteristics and age of onset of puberty were normal in all adult subjects studied. Consistent with these clinical findings, circulating levels of sex steroids and gonadotropins were appropriate for Tanner stage in all affected subjects (Table 3 ). Of note, the absence of clinical and biochemical signs of puber- ty in some subjects with markedly advanced bone age was surprising and suggests that the advance in bone age in these cases was being driven by something other than sex steroids.

Hyperinsulinemia, but not diabetes, has been described in the Mc4r-KO mouse. Congruent with this, none of the affected subjects in this study were hyperglycemic. Fasting insulin levels were elevated in all subjects when compared with similarly obese subjects matched for age (Table 3; I.S. Farooqi, unpublished observations), with four of the homozygous N62S children having markedly elevated fasting plasma insulin values (185-437 pmol/L). In none of these cases were proinsulin or split proinsulin levels excessively high, which contrasts with murine and human obesity syndromes associated with defects in carboxypeptidase or prohormone convertase $1(4,5)$. Plasma leptin levels appeared to reflect fat mass (Table 3).

The fact that all subjects with heterozygous frameshift mutations in $M C 4 R$ had severe early-onset obesity whereas heterozygotes for the N62S mutation were not obese was striking. To explore the molecular basis for this phenotypic heterogeneity, vectors expressing wildtype, CTCTA, GTins, N62S, and C271Y were transiently transfected into HEK293 cells. The cells were then
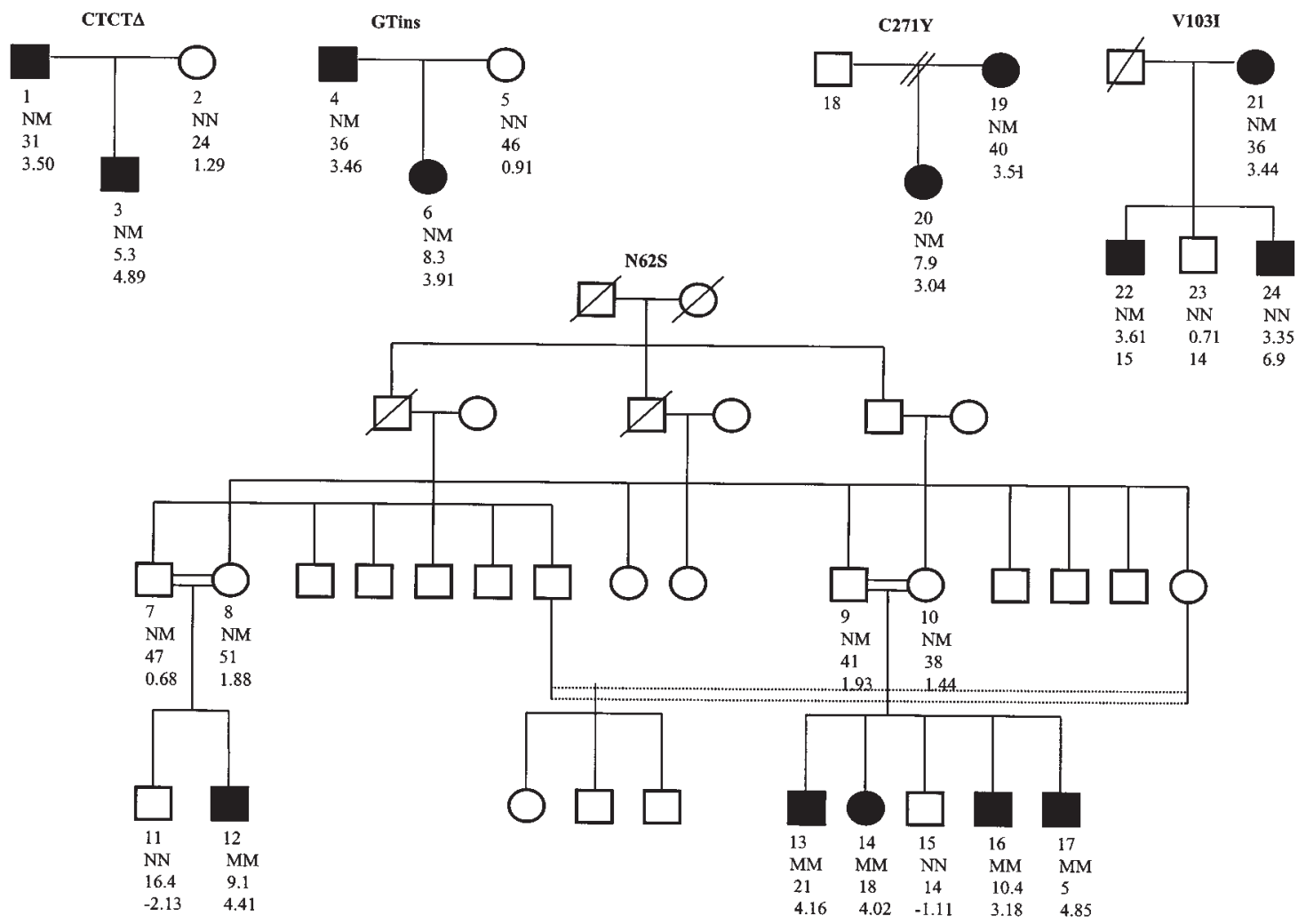

Figure 2

Inheritance of mutations in MC4R. Cosegregation of mutational status with BMI is shown for the five families in the mutational screening study for whom family data were available. Also shown is the family tree for the family with the CTCT deletion reported previously by us (9). Filled symbols represent subjects with severe obesity. The first line of the symbols corresponds to the identification number. The second line shows the genotype: N, normal allele; M, mutant allele. The third and fourth lines show current age of subject (years) and BMI standard deviation score, respectively. 
exposed to varying concentrations of $\alpha \mathrm{MSH}$, and the generation of cAMP was assayed indirectly through activation of a cotransfected cAMP-responsive reporter construct expressing luciferase (Figure 3). Cells expressing wild-type receptor showed a sigmoidal dose response to $\alpha \mathrm{MSH}$. In contrast, cells expressing the CTCT $\Delta$, GTins, and $\mathrm{C} 271 \mathrm{Y}$ receptors showed no response. Cells expressing the N62S receptor showed a response intermediate between the wild-type and nonsense mutant receptors.

\section{Discussion}

We determined the nucleotide sequence of the coding region of $M C 4 R$ in 243 unrelated probands with severe early-onset obesity and found seven probands with mutations that alter the amino acid sequence of the receptor and that are absent in 108 control alleles. Two previously reported conservative changes, V103I and $1251 \mathrm{~L}$, were found at similar frequencies in the case and control groups. T112M has also been reported previously in both obese and nonobese individuals $(11,12)$ and in one family studied by us. The significance of the alteration in the 3 -untranslated region in one subject is, as yet, unclear. Thus, the frequency of potentially pathogenic mutations in this series is

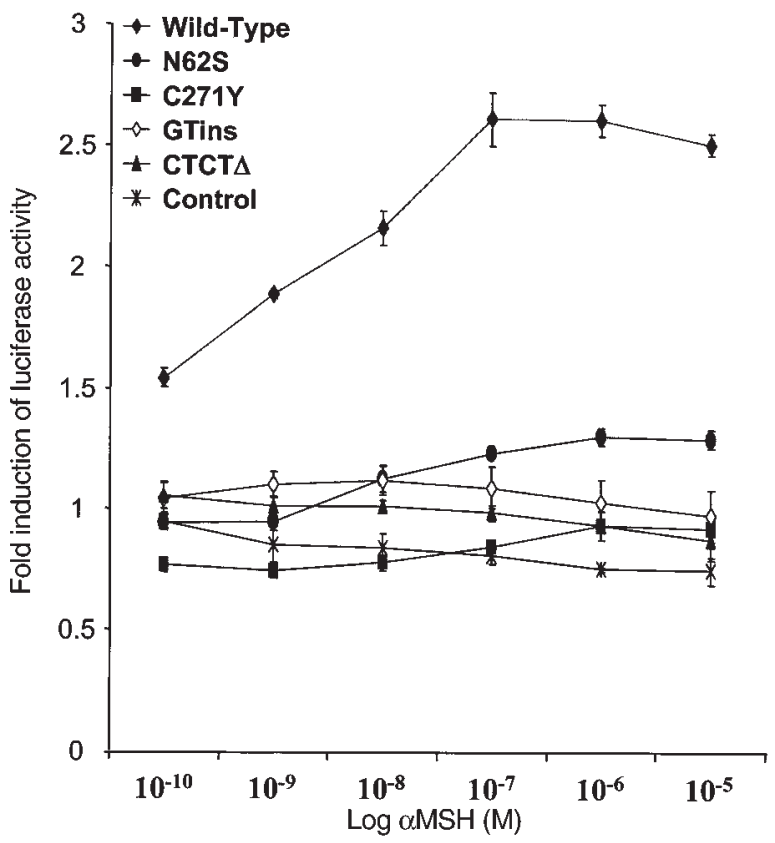

Figure 3

Signaling properties of mutant MC4Rs, N62S, GTins, CTCT $\Delta$, and C271Y. HEK293 cells were transiently transfected with either empty pcDNA3 vector or the same vector expressing wild-type, CTCT $\Delta$, GTins, N62S, and C271Y-mutant MC4Rs. The response to increasing concentrations of ligand $(\alpha M S H)$ was assessed by cotransfection with a cAMP-responsive reporter plasmid as described previously (18) and by measuring luciferase activity in a luminometer (see Methods). Data obtained were normalized for transfection efficiency by cotransfection of an internal control plasmid, pRL-CMV, which constitutively expresses Renilla luciferase. Data is expressed as a fold induction of luciferase activity. Each point represents the mean $( \pm S E)$ of at least three independent experiments performed in quadruplicate.
$3.3 \%$. Two other groups have reported similar studies. Gu et al. (11) screened MC4R in 140 relatively unselected obese subjects by single-strand conformational polymorphism (SSCP) analysis and reported two heterozygous missense mutations, V103I and I137T. In addition, a heterozygous missense mutation T112M was found in a lean individual. Functional analysis of these three mutations showed that the response of I137T to $\alpha \mathrm{MSH}$ was significantly attenuated, whereas the signaling properties of V103I and T112M were indistinguishable from wild-type. Hinney et al. reported nine missense mutations and two nonsense mutations in 306 obese children (12). These were a CTCT deletion at codon 211, as reported previously (9); a nonsense (Y35stop) and a missense mutation $(\mathrm{N} 37 \mathrm{~V})$, which form a haplotype that was found in two unrelated kindreds; S30F and G252S, which were found as a compound heterozygote in a single patient; and four heterozygous missense mutations P78L, T112M, R165W, and I317T. In addition, a silent mutation (V193V), as well as V103I and I251L, were reported. Subsequently, Ho and MacKenzie have shown that the CTCT-deletion mutant is nonfunctional due to the lack of a sequence in the cytoplasmic tail of MC4R necessary for cell-surface targeting of the protein (19). Thus, $M C 4 R$ mutations appear to be an appreciable cause of severe early-onset obesity in several different populations. Mutations in this gene represent the most common cause of monogenic human obesity described to date.

To better define the phenotypic features of human MC4R deficiency, we have studied affected and unaffected subjects from three families in which there is clear cosegregation of MC4R mutations with early-onset obesity. In addition to the obesity, it is of note that the majority of affected subjects have tall stature (mean 91st percentile). This value may be an underestimate because five affected subjects are of Indian/Pakistani and the normative height percentile data available are derived from U.K. Caucasians. Of particular note was the markedly increased linear growth, as measured by growth velocity, seen in children homozygous for the N62S mutation compared with their wild-type siblings. Radiological evidence for advanced bone age was seen in four of five affected children. Childhood obesity is known to be associated with advanced epiphyseal maturation $(22,23)$, however, in our cohort of obese children, the mean advancement of bone age is 1.7 years (I.S. Farooqi, unpublished observations). The degree of advancement in the MC4R-mutant subjects exceeded this in most cases, suggesting some specificity of this association. However, we have also reported similar degrees of advancement of bone age in severe obesity associated with congenital leptin deficiency (24).

Detailed measurements of body composition in affected subjects confirmed the expected large increase in fat mass. Of note, however, is the fact that percentage of body fat in affected subjects (e.g., $49.7 \%$ in subject 6 and $41.2 \%$ in subject 17) was somewhat less than 
Table 2

Clinical features, anthropometry, body composition, and energy balance in affected and unaffected members of families with the CTCT $\Delta$, GT ins, and N62S MC4R mutations

\begin{tabular}{|c|c|c|c|c|c|c|c|c|c|c|c|c|c|c|c|c|c|}
\hline \multirow[b]{2}{*}{ ID no. } & \multicolumn{3}{|c|}{ CTCT $\Delta$} & \multicolumn{3}{|c|}{ GTins } & \multicolumn{11}{|c|}{ N62S } \\
\hline & 1 & 2 & 3 & 4 & 5 & 6 & 7 & 8 & 9 & 10 & 11 & 12 & 13 & 14 & 15 & 16 & 17 \\
\hline $\begin{array}{l}\text { Genotype } \\
\text { Gender } \\
\text { Age (yr) }\end{array}$ & $\begin{array}{l}\text { NM } \\
\text { M } \\
31\end{array}$ & $\begin{array}{l}\text { NN } \\
\text { F } \\
24\end{array}$ & $\begin{array}{l}\text { NM } \\
\text { M } \\
5.3\end{array}$ & $\begin{array}{l}\text { NM } \\
\text { M } \\
36\end{array}$ & $\begin{array}{c}\text { NN } \\
\text { F } \\
46\end{array}$ & $\begin{array}{c}\text { NM } \\
\text { F } \\
8.3\end{array}$ & $\begin{array}{l}\text { NM } \\
\text { M } \\
47\end{array}$ & $\begin{array}{l}\text { NM } \\
\text { F } \\
51\end{array}$ & $\begin{array}{l}\text { NM } \\
\text { M } \\
41\end{array}$ & $\begin{array}{l}\text { NM } \\
\text { F } \\
38\end{array}$ & $\begin{array}{c}\mathrm{NN} \\
\mathrm{M} \\
16.4\end{array}$ & $\begin{array}{c}\text { MM } \\
\mathrm{M} \\
9.1\end{array}$ & $\begin{array}{c}\text { MM } \\
\text { M } \\
21\end{array}$ & $\begin{array}{c}\text { MM } \\
\text { F } \\
18\end{array}$ & $\begin{array}{l}N N \\
M \\
14\end{array}$ & $\begin{array}{c}\text { MM } \\
\text { M } \\
10.4\end{array}$ & $\begin{array}{c}\text { MM } \\
\text { M } \\
5\end{array}$ \\
\hline Weight (kg) & 138 & 65 & 38.4 & 118.6 & 61 & 71 & 65.7 & 72 & 83.5 & 60 & 44 & 115.5 & 153.4 & 119.2 & 41 & 62.4 & 40.6 \\
\hline Height percentile & 91 & 9 & 91 & 25 & 9 & $>99.6$ & 2 & 9 & 9 & 0.4 & 9 & $>99.6$ & 75 & 25 & 25 & 91 & $>99.6$ \\
\hline $\mathrm{BMI}\left(\mathrm{kg} / \mathrm{m}^{2}\right)$ & 40 & 26 & 28 & 40 & 25 & 33 & 24.7 & 29 & 29.5 & 27 & 16 & 45.4 & 48.4 & 46.2 & 16.6 & 29.2 & 27.3 \\
\hline BMI SDS & 3.5 & 1.29 & 4.89 & 3.46 & 0.9 & 3.91 & 0.68 & 1.88 & 1.93 & 1.44 & -2.13 & 4.41 & 4.16 & 4.02 & -1.11 & 3.18 & 4.85 \\
\hline $\mathrm{SBP}(\mathrm{mmHg})$ & 138 & 117 & 103 & 114 & 114 & 123 & 161 & 170 & 141 & 144 & 110 & 138 & 138 & 147 & 119 & 118 & 102 \\
\hline $\mathrm{DBP}(\mathrm{mmHg})$ & 79 & 76 & 56 & 79 & 73 & 74 & 115 & 110 & 89 & 96 & 77 & 76 & 81 & 90 & 82 & 71 & 83 \\
\hline Bone age (yr) & & & 5.0 & & & 12 & & & & & 16 & 14 & & & 17 & 12.5 & 6.0 \\
\hline $\begin{array}{l}\text { Body composition } \\
\% \text { fat }\end{array}$ & 37.4 & 35.6 & 37.3 & 39.6 & 31.3 & 49.7 & 27.7 & 43.8 & 33.7 & 36.8 & 5.3 & 52.4 & NA & 51.5 & 6.3 & 43.6 & 41.2 \\
\hline$\%$ fat-free mass & 62.5 & 63.1 & 62.1 & 60.4 & 67.4 & 48.8 & 71.2 & 56.2 & 66.3 & 62.6 & 94.5 & 47.6 & NA & 48.5 & 92.9 & 55.3 & 59.2 \\
\hline $\mathrm{BMC}(\mathrm{kg})$ & 4.04 & 2.35 & 0.96 & 3.52 & 2.32 & 1.63 & 2.25 & 2.39 & 3.10 & 2.49 & 2.20 & 2.09 & NA & 2.89 & 2.06 & 1.84 & 1.13 \\
\hline $\operatorname{BMD}\left(\mathrm{g} / \mathrm{cm}^{2}\right)$ & 1.41 & 1.16 & 0.89 & 1.33 & 1.20 & 0.95 & 1.16 & 1.20 & 1.42 & 1.27 & 1.07 & 1.11 & NA & 1.37 & 1.12 & 1.07 & 0.99 \\
\hline BMD Z score & 2.50 & 0.6 & 1.50 & 1.50 & 1.1 & 1.7 & -0.7 & -0.2 & 2.5 & 1.8 & -0.6 & 3.2 & NA & 3.1 & 1.3 & 2.4 & 2.7 \\
\hline $\begin{array}{l}\text { Energy expenditure } \\
\text { BMR (MJ/day) }\end{array}$ & 10.21 & 15.85 & 5.0 & 8.52 & 5.0 & 6.21 & 5.98 & 5.1 & 5.72 & 4.21 & 5.9 & 9.1 & 9.52 & 7.32 & 4.26 & 5.9 & 4.28 \\
\hline Predicted BMR (MJ/day) & 10.50 & 5.90 & 4.6 & 9.50 & 5.7 & 6.8 & 6.4 & 5.7 & 7.2 & 5.2 & 5.6 & 9.4 & 10.2 & 7.8 & 5.3 & 6.3 & 4.8 \\
\hline Energy intake & & & & & & & & & & & & & & & & & \\
\hline Ad libitum meal (MJ) & & & 4.61 & & & 4.52 & & & & & 1.81 & 6.45 & & & 2.06 & 3.09 & 1.62 \\
\hline
\end{tabular}

ID no. refers to identification number used in family tree in Figure 2. Affected subjects are in bold. BMI is weight in kilograms divided by height in meters squared, and BMI SDS is the standard deviation score calculated using U.K. population data. BMC is the total body bone mineral content; and BMD is total body bone mineral density ( $Z$ score is derived from age- and gender-specific means). Values for predicted BMR were derived using the equations of Hayter and Henry for Caucasian adults (13) (subjects 1, 2, 4, 5) and by using equations for adult Indian males (14) (subjects 7, 9 and 13) and Indian females (15) (subjects 8, 10 and 14). The equations derived by Molnar et al. were used to predict BMR for all children studied (16). SBP, systolic blood pressure; DBP diastolic blood pressure.

that seen by us in subjects of a similar age with obesity due to congenital leptin deficiency $(59.2 \%$ and $57.1 \%$, respectively; ref. 24 and I.S. Farooqi, unpublished observations). Interestingly, $\mathrm{BMC}$ and $\mathrm{BMD}$ were markedly increased in affected subjects. This increase in total BMC in adult affected subjects was comparable to that reported previously in unselected obese adults (25). Affected children had values considerably greater than normal-weight children of the same age, height, gender, and pubertal stage $(26,27)$ and total BMD was increased in comparison to values obtained from studies of obese children (28). Prediction charts for BMC in children based on height and weight have been derived from studies of children weighing up to $50 \mathrm{~kg}$ (29). Only two of our affected children (subjects 3 and 17) can be assessed using these standards. For both, the actual measured BMC is considerably greater than that predicted on the basis of height and weight $(0.58 \mathrm{~g} / \mathrm{cm}$ vs. $0.96 \mathrm{~g} / \mathrm{cm}$ and $0.58 \mathrm{~g} / \mathrm{cm}$ vs. $1.13 \mathrm{~g} / \mathrm{cm})$. However, the radiological appearance of the bones of affected children and adults was normal in terms of cortical thickness and cancellous bone pattern, which suggests that the increased BMC and BMD seen in these obese subjects may reflect bone size.

Whereas hyperphagia is clearly documented in MC4R-deficient mice, no information is available regarding the relative roles of excess energy intake vs. diminished energy expenditure in the pathogenesis of obesity associated with human MC4R deficiency. In the Caucasian MC4R-deficient subjects, BMR did not devi- ate from that predicted on the basis of weight and gender alone, suggesting that the presence of mutations in $M C 4 R$ is not associated with a major deficit in basal energy expenditure. The affected subjects in the N62S family did appear to have a trend toward a negative deviation from predicted BMR; however, this was also seen for nonobese individuals in this family and needs to be viewed in the light of the relative paucity of normative data on BMR in this ethnic group.

In this study, the energy ingested at a test meal was greatly increased in affected children compared to their wild-type siblings. This was consistent with the reported food-seeking behavior of the affected children in the free-living situation. Anecdotally, the intensity and persistence of the food-seeking behavior that was witnessed was less in MC4R-deficient children compared with the children with congenital leptin deficiency we have reported previously (24). All four affected adult subjects, described intense feelings of hunger during childhood that became less pronounced in late teens.

In contrast with several other monogenic syndromes of human obesity, pubertal development and reproductive function appear to be unimpaired in MC4Rdeficient subjects. Thyroid function was normal in all subjects and the significance of the raised urinary free cortisol excretion in two of seven affected subjects is uncertain. There were no clinical features of glucocorticoid excess in these subjects. All obese subjects were euglycemic but were hyperinsulinemic with a normal ratio of proinsulin and $32 / 33$ split proinsulin to insulin. 
Table 3

Metabolic and endocrine measurements in affected and unaffected members of families with the CTCT $\Delta$, GTins, and N62S MC4R mutations

\begin{tabular}{|c|c|c|c|c|c|c|c|c|c|c|c|c|c|c|c|c|c|}
\hline \multirow[b]{2}{*}{$\begin{array}{l}\text { ID no. } \\
\text { Genotype }\end{array}$} & \multicolumn{3}{|c|}{ СТСТ $\Delta$} & \multicolumn{3}{|c|}{ GTins } & \multicolumn{11}{|c|}{ N62S } \\
\hline & $\begin{array}{c}1 \\
\text { NM }\end{array}$ & $\stackrel{2}{N N}$ & $\begin{array}{c}3 \\
\text { NM }\end{array}$ & $\begin{array}{c}4 \\
\text { NM }\end{array}$ & $\begin{array}{c}5 \\
\text { NN }\end{array}$ & $\begin{array}{c}6 \\
\text { NM }\end{array}$ & $\begin{array}{c}7 \\
\text { NM }\end{array}$ & $\begin{array}{c}8 \\
\text { NM }\end{array}$ & $\begin{array}{c}9 \\
\text { NM }\end{array}$ & $\begin{array}{l}10 \\
\text { NM }\end{array}$ & $\begin{array}{l}11 \\
\text { NN }\end{array}$ & $\begin{array}{c}12 \\
\text { MM }\end{array}$ & $\begin{array}{c}13 \\
\text { MM }\end{array}$ & $\begin{array}{c}14 \\
\mathrm{MM}\end{array}$ & $\begin{array}{l}15 \\
\text { NN }\end{array}$ & $\begin{array}{c}16 \\
\text { MM }\end{array}$ & $\begin{array}{c}17 \\
\text { MM }\end{array}$ \\
\hline Gender & M & $\mathrm{F}$ & M & M & $\mathrm{F}$ & $\mathrm{F}$ & M & $\mathrm{F}$ & M & $\mathrm{F}$ & M & M & M & $\mathrm{F}$ & $\mathrm{M}$ & M & M \\
\hline Age $(y r)$ & 31 & 24 & 5.3 & 36 & 46 & 8.3 & 47 & 51 & 41 & 38 & 16.4 & 9.1 & 21 & 18 & 14 & 10.4 & 5 \\
\hline \multirow{5}{*}{$\begin{array}{l}\text { Metabolic status } \\
\text { Glucose (3.5-5.5 mmol/L) } \\
\text { Insulin (pmol/L) } \\
\text { Proinsulin (pmol/L) } \\
\text { Split proinsulin (pmol/L) } \\
\text { Leptin (ng/mL) }\end{array}$} & 5.6 & NA & 4.8 & NA & NA & & & & 5.0 & 4 & 4.5 & & & 2 & 47 & & 4.4 \\
\hline & 75 & 18 & $90^{A}$ & 152 & 29 & $134^{\mathrm{A}}$ & 85 & 82 & 132 & 71 & 24 & $437^{\mathrm{A}}$ & 408 & 210 & 28 & $185^{\mathrm{A}}$ & $87^{\mathrm{A}}$ \\
\hline & 4.3 & 1.4 & 1.9 & 4.4 & 2.5 & 9.1 & 6.1 & 16 & 12 & 2.8 & 2.0 & 15 & 19 & 46 & 3.7 & 18 & 6.1 \\
\hline & 6.7 & 1.25 & 4.7 & 6.6 & 3.7 & 22 & 12 & 20 & 18 & 5.8 & 1.8 & 36 & 30 & 37 & 4.9 & 45 & 12 \\
\hline & 13 & 9 & 18 & 18 & 15 & 34 & 12 & 42 & 17 & 18 & 1.8 & 70 & 52 & 98 & 1.6 & 15 & 9 \\
\hline \multicolumn{18}{|l|}{ Endocrine status } \\
\hline $\mathrm{TSH}(0.4-4.0 \mathrm{mU}$ & 2.10 & 0.70 & 4.30 & 1.60 & 0.97 & 6.1 & 1.30 & 0.42 & 0.99 & 0.97 & 1.00 & 3.30 & 6.0 & 2.90 & 3.30 & 1.70 & 2.60 \\
\hline Free thyroxine $(9-20 \mathrm{pmol} / \mathrm{L})$ & 12.6 & 18.3 & 14.6 & 11.4 & 14.7 & 14.3 & 9.1 & 15.9 & 12.4 & 11.1 & 15.8 & 12.3 & 10.6 & 15.5 & 13.1 & 12.7 & 13.3 \\
\hline АCTH $(5-50 \mathrm{ng} / \mathrm{L})$ & 19 & NA & 30 & 12 & 24 & 22 & 21 & 41 & 26 & 38 & 16 & 58 & 19 & 31 & NA & 14 & 27 \\
\hline 24-h UFC $(<200 \mathrm{nmol})$ & 274 & NA & NA & 331 & 190 & 116 & 133 & 148 & 669 & 114 & 218 & NA & 381 & 166 & 54 & 179 & 57 \\
\hline $\mathrm{LH}(\mathrm{mU} / \mathrm{L})$ & 5.7 & 6.2 & $<0.2$ & 3.1 & 14.8 & $<0.2$ & 6.5 & 41.1 & 3.1 & 4.0 & 1.6 & $<0.2$ & 4.8 & 5.7 & 1.8 & $<0.2$ & $<0.2$ \\
\hline $\mathrm{FSH}(\mathrm{mU} / \mathrm{L})$ & 3.2 & 9.3 & $<0.2$ & 3.2 & 40.8 & $<0.2$ & 12.5 & 72.8 & 3.2 & 4.2 & 2.8 & $<0.2$ & 2.4 & 4.1 & 1.4 & 1.4 & $<0.2$ \\
\hline Estradiol (pmol/L) & & 143 & & & 206 & 64 & & 71 & & 75 & & & & 186 & & & \\
\hline estosterone $(\mathrm{nmol} / \mathrm{L})$ & 23 & & $<0.2$ & 16 & & & 15 & & 16 & & 11 & $<0.2$ & 16.0 & & 36 & $<0.2$ & $<0.2$ \\
\hline
\end{tabular}

ID no. refers to identification number used in family tree in Figure 2. Affected subjects are in bold. Normal ranges for insulin $(0-60$ pmol/L), proinsulin $(<5$ $\mathrm{pmol} / \mathrm{L})$, and $32 / 33$ split proinsulin $(<13 \mathrm{pmol} / \mathrm{L}$ ) are based on values for lean healthy subjects. Normal ranges for $\mathrm{LH}$ : for men, 1.5-6.3 mU/L; for women in the follicular phase of the menstrual cycle, 1.3-8.4 mU/L, and for postmenopausal women, 16-75 mU/L. Normal ranges for FSH: for men 1.0-10.0 mU/L, for women in the follicular phase of the menstrual cycle, 2.9-8.4 mU/L, and for postmenopausal women, 21-140 mU/L. Normal ranges for testosterone for men, $8-38 \mathrm{nmol} / \mathrm{L}$. Normal range of estradiol for women in the follicular phase of the menstrual cycle, $100-750 \mathrm{pmol} / \mathrm{L}$, and for postmenopausal women, $<110$ $\mathrm{pmol} / \mathrm{L}$. ${ }^{A}$ Mean plasma insulin $(\mathrm{pmol} / \mathrm{L}) \pm \mathrm{SD}$ of age-matched obese children: for subject $3,36 \pm 16$; subject $6,70 \pm 30$; subject 12, $131 \pm 56$; subject 16,90 \pm 42 ; subject $17,36 \pm 16$. TSH, thyroid-stimulating hormone; 24 -h UFC, 24-hour urine free cortisol.

Interestingly the degree of hyperinsulinemia appears to be more severe in children and less marked in affected adults. This finding parallels the apparent amelioration of the intensity of hyperphagia that seems to occur with the attainment of adulthood in these subjects.

Blood pressure of affected children appears elevated with respect to age and gender-specific normative data (30), but three of five children have normal systolic and diastolic blood pressures $(<90$ th percentile) when adjusted for height, which is the most powerful determinant of blood pressure changes during childhood (31). Longitudinal studies in childhood and adolescence indicate that blood pressure parallels skeletal maturation more closely than chronological age (32), and it is therefore noteworthy that the two affected children with systolic blood pressures greater than the 95th percentile for age, gender, and height (subjects 6 and 12) have the most accelerated bone age.

The N62S mutation described in this study is unique, being the only $M C 4 R$ mutation described to date associated with a recessive, rather than dominant, pattern of inheritance of severe obesity. All four heterozygous carriers for N62S are nonobese, whereas the five homozygous children have severe early-onset obesity. Study of the signaling properties of this mutant receptor provides a possible explanation for this because, in contrast with the nonsense and missense mutations associated with dominantly inherited obesity, N62S retains some capacity to signal to cAMP generation. Thus, heterozygous carriers of this mutation will express one normal and one partially functioning allele, and this appears to be sufficient to prevent the development of severe obesity. Having said that, all four heterozygous carriers are overweight $\left(\mathrm{BMI}>25 \mathrm{~kg} / \mathrm{m}^{2}\right)$, and some give a history of excessive appetite in childhood, facts that may suggest that body-weight regulation is exquisitely sensitive to relatively subtle variation in the amount of functional MC4R expressed. Whereas a dominant-negative effect of the heterozygous mutations cannot as yet be formally excluded, it appears likely, given the known biology of G protein-coupled receptors, that heterozygous frameshift mutations result in a severe phenotype through the phenomenon of haploinsufficiency. A large number of inherited human endocrine disorders associated with loss of function mutations in $G$ protein-coupled receptors have now been described (33). In the majority of these disorders inheritance is typically recessive, with no clinical manifestations in heterozygotes. In this respect human MC4R deficiency most closely resembles familial hypocalciuric hypercalcemia due to mutations in the calcium-sensing receptor (34) in the preponderance of families with a dominant rather than recessive mode of inheritance.

In summary, potentially pathogenic mutations in the MC4R gene were found at a frequency of approximately $3-4 \%$ in severe early-onset obesity and represent the most common monogenic defect causing human obesity thus far described. Whereas dominant inheritance of obesity is the most typical pattern, recessive patterns of inheritance can occur, and this may reflect the specific effects of particular mutations on the functional properties of the mutant receptor. The human MC4Rdeficient phenotype closely resembles the murine-deficiency state in the preservation of reproductive function, the presence of hyperphagia, increased linear 
growth, and hyperinsulinemia and the absence of diabetes. Although the numbers of subjects involved are too small to permit formal comparison, the intensity of hyperphagia and the increase in percentage of body fat appear to be somewhat less in MC4R-deficient human subjects than those found in leptin-deficient subjects. The increase in BMD and advancement of bone age in MC4R-deficient subjects is worthy of further exploration, as is the possibility that the severity of the hyperphagia and hyperinsulinemia may lessen spontaneously with the attainment of adulthood. The detailed study of humans with morbid obesity due to specific monogenic defects should continue to shed light on the molecular mechanisms underlying the regulation of human appetite, body weight, and growth.

\section{Acknowledgments}

This work is supported by a Programme Grant from the Medical Research Council of the U.K. (S. O'Rahilly). I.S. Farooqi is a Wellcome Trust Training Fellow. We would like to thank the patients for their cooperation with this study and Uta Lange, Sally Brading, Maisam Fazel, and Jonathon Bull (medical students) for assistance with clinical studies, and N.J. Wareham for access to control populations. We thank Philip Bearcroft, Addenbrooke's Hospital, for assessment of bone age and all the physicians that have referred patients for our studies. We thank Merck Pharmaceuticals for an unconditional grant to assist the undertaking of clinical studies.

1. Yaswen, L., Diehl, N., Brennan, M.B., and Hochgeschwender, U. 1999. Obesity in the mouse model of pro-opiomelanocortin deficiency responds to peripheral melanocortin. Nat. Med. 5:1066-1070.

2. Krude, H., et al. 1998. Severe early-onset obesity, adrenal insufficiency and red hair pigmentation caused by POMC mutations in humans. Nat. Genet. 19:155-157.

3. Ollmann, M.M., et al. 1997. Antagonism of central melanocortin receptors in vitro and in vivo by agouti-related protein. Science. 278:135-138.

4. Naggert, J.K., et al. 1995. Hyperproinsulinaemia in obese fat/fat mice associated with a carboxypeptidase $\mathrm{E}$ mutation which reduces enzyme activity. Nat. Genet. 10:135-142.

5. Jackson, R.S., et al. 1997. Obesity and impaired prohormone processing associated with mutations in the human prohormone convertase 1 gene. Nat. Genet. 16:303-306.

6. Gantz, I., et al. 1993. Molecular cloning of a novel melanocortin receptor. J. Biol. Chem. 268:8246-8250.

7. Mountjoy, K.G., Mortrud, M.T., Low, M.J., Simerly, R.B., and Cone, R.D. 1994. Localization of the melanocortin-4 receptor (MC4-R) in neuroendocrine and autonomic control circuits in the brain. Mol. Endocrinol. 8:1298-1308.

8. Huszar, D., et al. 1997. Targeted disruption of the melanocortin-4 receptor results in obesity in mice. Cell. 88:131-141.

9. Yeo, G.S., et al. 1998. A frameshift mutation in MC4R associated with dominantly inherited human obesity. Nat. Genet. 20:111-112.

10. Vaisse, C., Clement, K., Guy-Grand, B., and Froguel, P. 1998. A frameshift mutation in human MC4R is associated with a dominant form of obesity. Nat.Genet. 20:113-114.
11. Gu, W., et al. 1999. Identification and functional analysis of novel human melanocortin-4 receptor variants. Diabetes. 48:635-639.

12. Hinney, A., et al. 1999. Several mutations in the melanocortin-4 receptor gene including a nonsense and a frameshift mutation associated with dominantly inherited obesity in humans. J. Clin. Endocrinol. Metab. 84:1483-1486.

13. Hayter, J.E., and Henry, C.J. 1994. A re-examination of basal metabolic rate predictive equations: the importance of geographic origin of subjects in sample selection. Eur. J. Clin. Nutr. 48:702-707.

14. Soares, M.J., Francis, D.G., and Shetty, P.S. 1993. Predictive equations for basal metabolic rates of Indian males. Eur. J. Clin. Nutr. 47:389-394.

15. Piers, L.S., and Shetty, P.S. 1993. Basal metabolic rates of Indian women. Eur. J. Clin. Nutr. 47:586-591.

16. Molnar, D., Jeges, S., Erhardt, E., and Schutz, Y. 1995. Measured and predicted resting metabolic rate in obese and nonobese adolescents. J. Pediatr. 127:571-577.

17. Holland, B., et al. 1991. McCance and Widdowson's the composition of foods. 5 th edition. Royal Society of Chemistry. Cambridge, United Kingdom. 290-883.

18. Persani, L., et al. 1993. Measurement of cAMP accumulation in Chinese hamster ovary cells transfected with the recombinant human TSH receptor (CHO-R): a new bioassay for human thyrotropin. $J$. Endocrinol. Invest. 16:511-519.

19. Ho, G., and MacKenzie, R.G. 1999. Functional characterization of mutations in melanocortin-4 receptor associated with human obesity. J. Biol. Chem. 274:35816-35822.

20. Maynard, L.M., et al. 1998. Total-body and regional bone mineral content and areal bone mineral density in children aged 8-18 y: the Fels Longitudinal Study. Am. J. Clin. Nutr. 68:1111-1117.

21. Henry, C.J., and Rees, D.G. 1991. New predictive equations for the estimation of basal metabolic rate in tropical peoples. Eur. J. Clin. Nutr. 45:177-185.

22. De Simone, M., et al. 1995. Growth charts, growth velocity and bone development in childhood obesity. Int. J. Obes. Relat. Metab. Disord. 19:851-857.

23. Polito, C., et al. 1995. Advanced RUS and normal carpal bone age in childhood obesity. Int. J. Obes. Relat. Metab. Disord. 19:506-507.

24. Faroogi, I.S., et al. 1999. Effects of recombinant leptin therapy in a child with congenital leptin deficiency. N. Engl. J. Med. 341:879-884.

25. Compston, J.E., Laskey, M.A., Croucher, P.I., Coxon, A., and Kreitzman, S. 1992. Effect of diet-induced weight loss on total body bone mass. Clin. Sci. 82:429-432.

26. Nelson, D.A., Simpson, P.M., Johnson, C.C., Barondess, D.A., Kleerekoper, M. 1997. The accumulation of whole body skeletal mass in third- and fourth-grade children: effects of age, gender, ethnicity, and body composition. Bone. 20:73-78.

27. Zanchetta, J.R., Plotkin, H., and Alvarez Filgueira, M.L. 1995. Bone mass in children: normative values for the 2-20 year old population. Bone. 16(Suppl. 4):393S-399S.

28. Manzoni, P., et al. 1996. Influence of body composition on bone mineral content in children and adolescents. Am. J. Clin. Nutr. 64:603-607.

29. Bishop, N.J., dePriester, J.A., Cole, T.J., and Lucas, A. 1992. Reference values for radial bone width and mineral content using single photon absorptiometry in healthy children aged 4 to 10 years. Acta Paediatr. 81:463-468.

30. 1996. Update on the 1987 Task Force Report on High Blood Pressure in Children and Adolescents: a working group report from the National High Blood Pressure Education Program. National High Blood Pressure Education Program Working Group on Hypertension Control in Children and Adolescents. Pediatrics. 98:649-658.

31. Rosner, B., Prineas, R.J., Loggie, J.M., and Daniels, S.R. 1993. Blood pressure nomograms for children and adolescents, by height, sex, and age, in the United States. J. Pediatr. 123:871-886.

32. Clarke, W.R., Woolson, R.F., and Lauer, R.M. 1986. Changes in ponderosity and blood pressure in childhood: the Muscatine Study. Am. J. Epidemiol. 124:195-206.

33. Spiegel, A.M. 1996. Defects in G protein-coupled signal transduction in human disease. Annu. Rev. Physiol. 58:143-170.

34. Spiegel, A.M. 1996. Mutations in G proteins and G protein-coupled receptors in endocrine disease. J. Clin. Endocrinol. Metab. 81:2434-2442. 